\title{
Extremal Myers-Perry black holes coupled to Born-Infeld electrodynamics in five dimensions
}

\author{
Masoud Allahverdizadeh ${ }^{1 *}$, José P. S. Lemos ${ }^{1 \dagger}$, Ahmad Sheykhi ${ }^{2,3 \ddagger}$ \\ 1 Centro Multidisciplinar de Astrofísica - CENTRA, \\ Departamento de Física, Instituto Superior Técnico - IST, \\ Universidade Técnica de Lisboa - Av. Rovisco Pais 1, 1049-001 Lisboa, Portugal. \\ 2 Physics Department and Biruni Observatory, \\ College of Sciences, Shiraz University, Shiraz 71454, Iran \\ ${ }^{3}$ Research Institute for Astronomy and Astrophysics of \\ Maragha (RIAAM), P.O. Box 55134-441, Maragha, Iran
}

\begin{abstract}
We construct a new class of perturbative extremal charged rotating black hole solutions in five dimensions in the Einstein-Born-Infeld theory. We start with an extremal fivedimensional Myers-Perry black hole seed in which the two possible angular momenta have equal magnitude, and add Born-Infeld electrical charge keeping the extremality condition as a constraint. The perturbative parameter is assumed to be the electric charge $q$ and the perturbations are performed up to 4 th order. We also study some physical properties of these black holes. It is shown that the perturbative parameter $q$ and the Born-Infeld parameter $\beta$ modify the values of the physical quantities of the black holes. The solution suggests that the magnetic moment and gyromagnetic ratio of the black hole spacetime change sign when the Born-Infeld character of the solution starts to depart strongly from the Maxwell limit. We venture an interpretation for the effect.
\end{abstract}

\section{INTRODUCTION}

The field of black hole solutions in higher dimensions was started in 1963 by Tangherlini [1]. After some discussion of the old problem of the dimensionality of space he proceed to find static spherical symmetric solutions of the $d$ dimensional Einstein-Maxwell equations, with $d \geq 4$, gener-

\footnotetext{
*masoud.alahverdi@gmail.com

† joselemos@ist.utl.pt

‡ asheykhi@shirazu.ac.ir
} 
alizing thus the Schwarzschild and the Reissner-Nordström solutions. Tangherlini also included a cosmological term finding the corresponding de Sitter (dS) and anti-de Sitter (AdS) $d$-dimensional black holes [1].

Since then, with the belief that at a more fundamental level Einstein's theory has to be modified and extra dimensions might be crucial in the process, static higher-dimensional black holes solutions have been devised in gravitational theories such as Kaluza-Klein, Gauss-Bonnet theory and its Lovelock extension, scalar-tensor gravity, supergravity, and low-energy string theory. Likewise, there has been attempts to modify Maxwell's electromagnetism in order to deal in a more consistent manner with the point particle problem and its field divergences. An important modification is given by the Born-Infeld nonlinear electrodynamics. Although it became less prominent with the appearance of quantum electrodynamics and its renormalization scheme, the Born-Infeld theory has been now occurring repeatedly with the development of string theory, where the dynamics of D-branes is governed by a Born-Infeld type action. It is thus natural to study higher dimensional black holes in these extended gravity-electromagnetic set ups.

For the static case, several examples of higher dimensional black holes can be given. Black holes in Lovelock gravity and Maxwell electromagnetism were studied in [2]. Black hole solutions in Einstein-Born-Infeld gravity are less singular in comparison with the Tangherlini-ReissnerNordström solution and such solutions with and without a cosmological constant have been discussed in general relativity [3 8], and in Gauss-Bonnet [9] and Lovelock [10] gravities. The extension to the cases where the horizon has zero or negative curvature has also been considered [11]. Scalar-tensor theories of gravity coupled to Born-Infeld black hole solutions have also been studied in [12]. Attempts to find solutions in general relativity coupled to other fields, such as dilaton, rank three antisymmetric tensor, and Born-Infeld and others, have been performed to construct exact solutions [13-16]. In these theories the dilaton field can be thought of as coming from a scalar field of low energy limit of string theory. The appearance of the dilaton field changes the asymptotic behavior of the solutions to be neither asymptotically flat nor de Sitter or Anti-de Sitter.

Introducing rotation makes the search for solutions much more difficult. Following the example of the extension of the Schwarzschild and Reissner-Nordström solutions to higher dimensions in different theories, it was natural to do the same for the Kerr and the Kerr-Newman solutions. The generalization of the Kerr solution to higher dimensional Einstein gravity was performed by Myers and Perry [17]. These Myers-Perry solutions include the non-trivial cases of several modes of rotation due to the existence of other rotation planes in higher dimensions. The inclusion of a cosmological constant on these higher dimensional solutions was performed in [18]. Rotating black 
hole solutions in other gravity theories like Gauss-Bonnet or Lovelock are not know.

Finally, the generalization of the Kerr-Newman black holes to higher dimensional EinsteinMaxwell theory has not been found. Likewise, the generalization of Kerr with some other gauge field, different from Maxwell, to higher dimensions has not had success. In order to study this problem one has to resort to approximations, as was done in [19], when studying charged rotating black hole solutions in higher dimensions with a single rotation parameter in the limit of slow rotation (see also [20 22]). Further study, relying on perturbative or numerical methods, to construct those solutions in asymptotically flat backgrounds has been performed in 23] and in asymptotically anti-de Sitter spacetimes in [24]. Employing higher perturbation theory with the electric charge as the perturbation parameter, solutions and properties of charged rotating Einstein-Maxwell asymptotically flat black holes have been constructed in five dimensions [25]. Focusing on extremal black holes, this perturbative method was also applied to obtain Einstein-Maxwell black holes with equal magnitude angular momenta in odd dimensions [26]. A generalization to include a scalar field in a Einstein-Maxwell-dilaton theory was performed in [27] where black holes with equal magnitude angular momenta in general odd dimensions were obtained.

In this paper, we use this perturbative approach to find extremal rotating Einstein-Born-Infeld black holes in a five dimensional spacetime. Starting from the Myers-Perry black holes [17], we evaluate the perturbative series up to 4 th order in the Born-Infeld electric charge parameter $q$. We determine the physical properties of these black holes for general Born-Infeld coupling constant $\beta$. In fact, we study the effects of the perturbative parameter $q$ and the Born-Infeld parameter $\beta$ on the mass, angular momentum, magnetic moment, gyromagnetic ratio, and horizon radius of these rotating black holes.

The outline of this paper is as follows: In section II, we present the basic field equations of nonlinear Born-Infeld theory in Einstein gravity and obtain a new class of perturbative charged rotating solutions in five dimensions. In section III we calculate the physical quantities of the solutions and discuss their properties. In section IV we study the mass formula for these black holes. Section $\square$ is devoted to conclusions. 


\section{METRIC AND GAUGE POTENTIAL}

We start with the Einstein-Hilbert action coupled to the Born-Infeld nonlinear gauge field in five dimensions

$$
S=\int d x^{5} \sqrt{-g}\left(\frac{R}{16 \pi G_{5}}+L(F)\right)
$$

where $G_{5}$ is five dimensional Newtonian constant, $R$ is the curvature scalar and $L(F)$ is the Lagrangian of the nonlinear Born-Infeld gauge field given by

$$
L(F)=4 \beta^{2}\left(1-\sqrt{1+\frac{F^{\mu \nu} F_{\mu \nu}}{2 \beta^{2}}}\right),
$$

where, in turn, $F_{\mu \nu}=\partial_{\mu} A_{\nu}-\partial_{\nu} A_{\mu}$ is the electromagnetic field tensor, $A_{\mu}$ is the electromagnetic vector potential, and $\beta$ is the Born-Infeld parameter with unit of mass. In the limit $\beta \rightarrow \infty, L(F)$ reduces to the Lagrangian of the standard Maxwell field, $L(F)=F^{\mu \nu} F_{\mu \nu}$. The equations of motion can be obtained by varying the action with respect to the gravitational field $g_{\mu \nu}$ and the gauge field $A_{\mu}$. This procedure yields the gravitational field equations

$$
G_{\mu \nu}=R_{\mu \nu}-\frac{1}{2} g_{\mu \nu} R=\frac{1}{2} g_{\mu \nu} L(F)+\frac{2 F_{\mu \eta} F_{\nu}^{\eta}}{\sqrt{1+\frac{F^{\mu \nu} F_{\mu \nu}}{2 \beta^{2}}}},
$$

and the electromagnetic equation

$$
\partial_{\mu}\left(\frac{\sqrt{-g} F^{\mu \nu}}{\sqrt{1+\frac{F^{\mu \nu} F_{\mu \nu}}{2 \beta^{2}}}}\right)=0
$$

Our aim here is to find perturbative extremal charged rotating black hole solutions of the above field equations in five dimensions. Using coordinates $\left(t, r, \theta, \varphi_{1}, \varphi_{2}\right)$, the 5-dimensional Myers-Perry solution [17] restricted to the case where the two possible angular momenta have equal magnitude, and following [25] for the parametrization of the metric, one has

$$
\begin{aligned}
d s^{2}= & g_{t t} d t^{2}+\frac{d r^{2}}{W}+r^{2}\left(d \theta^{2}+\sin ^{2} \theta d \varphi_{1}^{2}+\cos ^{2} \theta d \varphi_{2}^{2}\right)+N\left(\varepsilon_{1} \sin ^{2} \theta d \varphi_{1}+\varepsilon_{2} \cos ^{2} \theta d \varphi_{2}\right)^{2} \\
& -2 B\left(\varepsilon_{1} \sin ^{2} \theta d \varphi_{1}+\varepsilon_{2} \cos ^{2} \theta d \varphi_{2}\right) d t
\end{aligned}
$$

where $\varepsilon_{k}$ denotes the sense of rotation in the $k$-th orthogonal plane of rotation, such that $\varepsilon_{k}=$ $\pm 1, k=1,2$. For the mentioned Myers-Perry solution [17] one has $g_{t t}=-1+\frac{2 \hat{M}}{r^{2}}, W=1-$ $\frac{2 \hat{M}}{r^{2}}+\frac{2 \hat{J}^{2}}{\hat{M} r^{4}}$, and $N=\frac{2 \hat{J}^{2}}{\hat{M} r^{2}}$, where $\hat{M}$ and $\hat{J}$ are two constants, namely, the mass and angular momentum parameters, respectively, related to the mass $M$ and angular momenta $J$ of the MyersPerry solution, through the relations $\hat{M}=\frac{16 \pi G_{5}}{3 A} M$ and $\hat{J}=\frac{4 \pi G_{5}}{A} J$, where $A$ is the area of the unit 
3-sphere. An adequate parametrization for the gauge potential is given by

$$
A_{\mu} d x^{\mu}=a_{0}+a_{\varphi}\left(\varepsilon_{1} \sin ^{2} \theta d \varphi_{1}+\varepsilon_{2} \cos ^{2} \theta d \varphi_{2}\right) .
$$

We further assume the metric functions $g_{t t}, W, N, B$ and also the two functions $a_{0}, a_{\varphi}$ for the gauge field, depend only on the radial coordinate $r$.

We consider perturbations around the Myers-Perry solution, with a Born-Infeld electric charge $q$ as the perturbative parameter, so that $q$ is much less than $\hat{M}$ or $\hat{J}^{2 / 3}$. Taking into account the symmetry with respect to charge reversal and the seed solution, the metric and gauge potentials take the form

$$
\begin{gathered}
g_{t t}=-1+\frac{2 \hat{M}}{r^{2}}+q^{2} g_{t t}^{(2)}+q^{4} g_{t t}^{(4)}+O\left(q^{6}\right), \\
W=1-\frac{2 \hat{M}}{r^{2}}+\frac{2 \hat{J}^{2}}{\hat{M} r^{4}}+q^{2} W^{(2)}+q^{4} W^{(4)}+O\left(q^{6}\right), \\
N=\frac{2 \hat{J}^{2}}{\hat{M} r^{2}}+q^{2} N^{(2)}+q^{4} N^{(4)}+O\left(q^{6}\right), \\
B=\frac{2 \hat{J}}{r^{2}}+q^{2} B^{(2)}+q^{4} B^{(4)}+O\left(q^{6}\right), \\
a_{0}=q a_{0}^{(1)}+q^{3} a_{0}^{(3)}+O\left(q^{5}\right), \\
a_{\varphi}=q a_{\varphi}^{(1)}+q^{3} a_{\varphi}^{(3)}+O\left(q^{5}\right) .
\end{gathered}
$$

Here $g_{t t}^{(2)}$ and $g_{t t}^{(4)}$ are the second and fourth order perturbative terms, respectively. The other perturbative terms are defined similarly.

We now fix the angular momenta at any perturbative order, and impose the extremal condition in all orders. We also assume that the horizon is regular. With these assumptions we are able to fix all constants of integration. To simplify the notation we introduce a parameter $\nu$ through the equations

$$
\hat{M}=2 \nu^{2} \quad, \quad \hat{J}=2 \nu^{3}
$$

meaning that the extremal Myers-Perry solution holds in five dimensions. Then, using the field equations (3)-(4), the perturbative solutions up to 4 th order can be written as,

$$
\begin{aligned}
g_{t t}= & -1+\frac{4 \nu^{2}}{r^{2}}+\frac{\left(r^{2}-4 \nu^{2}\right) q^{2}}{3 \nu^{2} r^{4}}+\left\{\frac{11}{135} \frac{1}{\nu^{2} \beta^{2} r^{8}}-\frac{16}{45} \frac{\nu^{2}}{\beta^{2} r^{12}}+\frac{58}{135} \frac{1}{\beta^{2} r^{10}}\right. \\
& -\frac{8 \nu^{2} \beta^{2}+3}{9 \beta^{2} \nu^{6} r^{4}}+\frac{1}{540} \frac{79+240 \nu^{2} \beta^{2}}{\beta^{2} \nu^{4} r^{6}}+\frac{1}{720} \frac{79+220 \nu^{2} \beta^{2}}{\beta^{2} \nu^{8} r^{2}} \\
& \left.\frac{4\left(r^{2}-2 \nu^{2}\right)^{2}}{27 \beta^{2} \nu^{10} r^{4}}\left(\nu^{2} \beta^{2}+\frac{3}{8}\right) \ln \left(1-2 \frac{\nu^{2}}{r^{2}}\right)\right\} q^{4}+O\left(q^{6}\right),
\end{aligned}
$$




$$
\begin{aligned}
& W=1-\frac{4 \nu^{2}}{r^{2}}+\frac{4 \nu^{4}}{r^{4}}-\frac{q^{2}\left(r^{2}-2 \nu^{2}\right)}{3 \nu^{2} r^{4}} \\
& +\left\{\frac{1}{2160} \frac{522+480 \nu^{2} \beta^{2}}{\beta^{2} \nu^{10}}+\frac{1}{2160} \frac{-2420 \nu^{4} \beta^{2}-2607 \nu^{2}}{\beta^{2} \nu^{10} r^{2}}+\frac{1}{2160} \frac{3840 \nu^{4}+3620 \beta^{2} \nu^{6}}{\beta^{2} \nu^{10} r^{4}}\right. \\
& +\frac{1}{2160} \frac{-1032 \nu^{6}-1280 \nu^{8} \beta^{2}}{\beta^{2} \nu^{10} r^{6}}-\frac{1}{5} \frac{1}{r^{8} \nu^{2} \beta^{2}}-\frac{23}{45} \frac{1}{\beta^{2} r^{10}}+\frac{44}{45} \frac{\nu^{2}}{\beta^{2} r^{12}}-\frac{56}{45} \frac{\nu^{4}}{\beta^{2} r^{14}} \\
& \left.+\frac{\left(r^{2}-2 \nu^{2}\right)^{3}}{9 \beta^{2} \nu^{12} r^{4}}\left(\frac{87}{80}+\nu^{2} \beta^{2}\right) \ln \left(1-2 \frac{\nu^{2}}{r^{2}}\right)\right\} q^{4}+O\left(q^{6}\right) \\
& N=\frac{4 \nu^{4}}{r^{2}}-\frac{2 q^{2}\left(r^{2}+2 \nu^{2}\right)}{3 r^{4}}+\left\{-\frac{1}{360} \frac{\left(87+80 \nu^{2} \beta^{2}\right) r^{2}}{\beta^{2} \nu^{10}}+\frac{52}{135} \frac{\nu^{2}}{\beta^{2} r^{10}}+\frac{4}{135} \frac{1}{\beta^{2} r^{8}}-\frac{16}{45} \frac{\nu^{4}}{\beta^{2} r^{12}}\right. \\
& +\frac{1}{45} \frac{1+20 \nu^{2} \beta^{2}}{r^{6} \nu^{2} \beta^{2}}-\frac{1}{90} \frac{61+40 \nu^{2} \beta^{2}}{\beta^{2} \nu^{4} r^{4}}+\frac{1}{360} \frac{87+80 \nu^{2} \beta^{2}}{\nu^{8} \beta^{2}}+\frac{1}{180} \frac{144+35 \nu^{2} \beta^{2}}{\beta^{2} \nu^{6} r^{2}} \\
& \left.-\ln \left(1-2 \frac{\nu^{2}}{r^{2}}\right)\left(\frac{87}{80} r^{6}+r^{6} \nu^{2} \beta^{2}-\frac{57}{20} \nu^{4} r^{2}+\nu^{6}+\frac{8}{3} \nu^{8} \beta^{2}\right) \frac{\left(r^{2}-2 \nu^{2}\right)}{9 \beta^{2} \nu^{12} r^{4}}\right\} q^{4}+O\left(q^{6}\right), \\
& B=\frac{4 \nu^{3}}{r^{2}}-\frac{4 \nu}{3 r^{4}} q^{2}+\left\{\frac{1}{1440} \frac{480 \nu^{2} \beta^{2}+864}{\beta^{2} \nu^{7} r^{2}}+\frac{2}{27} \frac{1}{\beta^{2} \nu r^{8}}+\frac{52}{135} \frac{\nu}{\beta^{2} r^{10}}-\frac{1}{60} \frac{29+40 \nu^{2} \beta^{2}}{\beta^{2} \nu^{5} r^{4}}\right. \\
& +\frac{1}{90} \frac{9+40 \nu^{2} \beta^{2}}{\beta^{2} \nu^{3} r^{6}}-\frac{16}{45} \frac{\nu^{3}}{\beta^{2} r^{12}}-\frac{1}{45} \frac{9+5 \nu^{2} \beta^{2}}{\beta^{2} \nu^{9}}+\left(-\frac{1}{270} \frac{27+15 \nu^{2} \beta^{2}}{\beta^{2} \nu^{11}}\right. \\
& \left.\left.+\frac{1}{270} \frac{54 \nu^{2}+30 \nu^{4} \beta^{2}}{\beta^{2} \nu^{11} r^{2}}-\frac{1}{270} \frac{80 \beta^{2} \nu^{6}+30 \nu^{4}}{\beta^{2} \nu^{11} r^{4}}\right)\left(r^{2}-2 \nu^{2}\right) \ln \left(1-2 \frac{\nu^{2}}{r^{2}}\right)\right\} q^{4}+O\left(q^{6}\right), \\
& a_{0}=\frac{q}{r^{2}}+\left\{\frac{1}{180} \frac{40 \nu^{2} \beta^{2}+15}{\beta^{2} \nu^{6} r^{2}}-\frac{1}{180} \frac{15 \nu^{2}+40 \nu^{4} \beta^{2}}{\beta^{2} \nu^{6} r^{4}}-\frac{11}{180} \frac{1}{r^{6} \nu^{2} \beta^{2}}-\frac{26}{45} \frac{1}{\beta^{2} r^{8}}+\frac{2}{3} \frac{\nu^{2}}{\beta^{2} r^{10}}\right. \\
& \left.+\frac{1}{9}\left(\nu^{2} \beta^{2}+\frac{3}{8}\right)\left(r^{2}-2 \nu^{2}\right) \ln \left(1-2 \frac{\nu^{2}}{r^{2}}\right) \beta^{-2} \nu^{-8} r^{-2}\right\} q^{3}+O\left(q^{5}\right), \\
& a_{\varphi}=-\frac{\nu q}{r^{2}}+\left\{-\frac{1}{720} \frac{80 \nu^{2} \beta^{2}+30}{\nu^{7} \beta^{2}}-\frac{1}{720} \frac{40 \nu^{4} \beta^{2}+27 \nu^{2}}{\nu^{7} \beta^{2} r^{2}}-\frac{1}{720} \frac{-160 \beta^{2} \nu^{6}-88 \nu^{4}}{\nu^{7} \beta^{2} r^{4}}+\frac{7}{60} \frac{1}{\nu \beta^{2} r^{6}}\right. \\
& \left.+\frac{26}{45} \frac{\nu}{\beta^{2} r^{8}}-\frac{2}{3} \frac{\nu^{3}}{\beta^{2} r^{10}}-\frac{1}{144}\left(3+8 \nu^{2} \beta^{2}\right)\left(\frac{r^{2}}{\beta^{2} \nu^{9}}-\frac{4}{r^{2} \beta^{2} \nu^{5}}\right) \ln \left(1-2 \frac{\nu^{2}}{r^{2}}\right)\right\} q^{3}+O\left(q^{5}\right) .
\end{aligned}
$$

It is seen that there are the usual $1 / r^{n}$ polynomial expressions as well as terms involving logarithmic functions. It is also worth mentioning that the Born-Infeld parameter $\beta$ appears in terms which are of 3rd and 4th order in the electric charge parameter q. One may note that in Maxwell's limit, $\beta \longrightarrow \infty$, these perturbative solutions reduce to the five dimensional perturbative charged rotating black holes in Einstein-Maxwell theory presented in [25]. A consistent check of these solutions can be provided by Smarr's formula. 


\section{PHYSICAL QUANTITIES}

The mass $M$, the angular momenta $J$, the electric charge $Q$, and the magnetic moment $\mu_{\text {mag }}$ can be read off the asymptotic behavior of the metric and the gauge potential [23]. The asymptotic forms are,

$$
g_{t t}=-1+\frac{\tilde{M}}{r^{2}}+\ldots, \quad B=\frac{2 \tilde{J}}{r^{2}}+\ldots, \quad a_{0}=\frac{\tilde{Q}}{r^{2}}+\ldots, \quad a_{\varphi}=\frac{\tilde{\mu}_{\mathrm{mag}}}{r^{2}}+\ldots
$$

where $\tilde{M}, \tilde{J}, \tilde{Q}$, and $\tilde{\mu}_{\text {mag }}$ are the mass, angular momentum, electric charge, and magnetic moment parameters, respectively, and we have defined $\tilde{Q} \equiv q$ for notational consistency. These parameters are related to the real mass $M$, angular momentum $J$, electric charge $Q$, and magnetic moment $\mu_{\text {mag }}$, through the relations,

$$
\begin{aligned}
& \tilde{M}=\frac{16 \pi G_{5}}{3 A} M, \quad \tilde{J}=\frac{4 \pi G_{5}}{A} J \\
& \tilde{Q}=\frac{4 \pi G_{5}}{2 A} Q, \quad \tilde{\mu}_{\mathrm{mag}}=\frac{4 \pi G_{5}}{2 A} \mu_{\mathrm{mag}}
\end{aligned}
$$

Note that when the perturbative parameter $q$ is equal to zero, the tilde quantities of Eq. (20) reduce to the hat quantities of Eq. (13), i.e., $\tilde{M}=\hat{M}, \tilde{J}=\hat{J}, \tilde{Q}=\hat{Q}=0$, and $\tilde{\mu}_{\text {mag }}=\hat{\mu}_{\text {mag }}=0$.

Now, comparing the expansions in Eqs. (20) and Eq. (21) with the asymptotic behavior of the solutions given in Eqs. (14)-(19), we obtain

$$
\begin{gathered}
M=\frac{3 \pi \nu^{2}}{2}+\frac{\pi q^{2}}{8 \nu^{2}}+\frac{\pi q^{4}\left(20 \nu^{2} \beta^{2}-3\right)}{5760 \beta^{2} \nu^{8}}+O\left(q^{6}\right), \\
J=\pi \nu^{3}, \\
Q=\pi q, \\
\mu_{\mathrm{mag}}=\pi \nu q-\frac{\pi q^{3}\left(40 \nu^{2} \beta^{2}+3\right)}{720 \nu^{5} \beta^{2}}+O\left(q^{5}\right),
\end{gathered}
$$

The gyromagnetic ratio $g$ is then given by

$$
g=2 \frac{\mu_{\mathrm{mag}} / Q}{J / M}=\frac{2 M \mu_{\mathrm{mag}}}{Q J}=3+\frac{q^{2}\left(20 \nu^{2} \beta^{2}-3\right)}{240 \nu^{6} \beta^{2}}-\frac{q^{4}\left(10 \nu^{2} \beta^{2}+3\right)}{1440 \nu^{10} \beta^{2}}+O\left(q^{6}\right) .
$$

The horizon radius $r_{H}$ is given by

$$
r_{H}=\sqrt{2} \nu+\frac{q^{2} \sqrt{2}}{24 \nu^{3}}+\frac{11}{1152} \frac{q^{4} \sqrt{2}}{\nu^{7}}+O\left(q^{6}\right) .
$$

All these quantities are worth commenting. 
The mass $M$ of the black holes as a function of the Born-Infeld parameter $\beta$ has an interesting behavior, as shown in Fig. 1, see also Eq. (22). The mass $M$ increases with increasing $\beta$ and as $\beta \rightarrow \infty$, i.e., in the Maxwell limit, the mass takes the value $M=\frac{3 \pi \nu^{2}}{2}+\frac{\pi q^{2}}{8 \nu^{2}}+\frac{\pi q^{4}}{288 \nu^{6}}+O\left(q^{6}\right)$, exactly the result obtained for the five-dimensional perturbative Einstein-Maxwell black hole [25]. For very small $\beta$ the mass turns negative. We do not attach any significance to this result since the value of $\beta$ for which the mass is zero uses a perturbative $q^{2}$ term which is much larger than than the zeroth order term in the expressions for the magnetic moment and gyromagnetic ratio. In fact, the values of $\beta$ for which the results make thorough sense are values of $\beta$ larger than the ones which yield the zeros of the magnetic moment and gyromagnetic ratio.

The angular momenta $J$ in Eq. (23) and the charge $q$ in Eq. (24) are fixed and do not depend

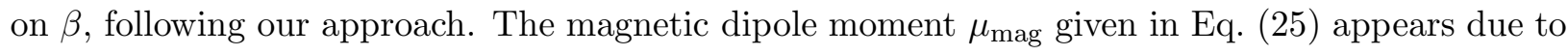
the rotation of the electrically charged black hole. The first order term $\pi q \nu=Q \nu$ is equivalent to the magnetic moment of a point particle rotating around an axis, with charge $q_{\text {particle }}=3 \pi Q$, and with the same angular momentum $j_{\text {particle }}$ and mass $m_{\text {particle }}$ of the black hole, i.e., $j_{\text {particle }}=J$ and $m_{\text {particle }}=M$, since for such a system $\mu_{\text {mag }}=\frac{1}{2} q_{\text {particle }} \frac{j_{\text {particle }}}{m_{\text {particle }}}=Q \nu$. The higher order terms presumably come from the spacetime curvature. From Fig. 2 we find out that the magnetic dipole moment $\mu_{\text {mag }}$ increases with increasing $\beta$. In the limit $\beta \rightarrow \infty$, the mass, the magnetic moment $\mu_{\mathrm{mag}}=\pi \nu q-\frac{\pi q^{3}}{18 \nu^{3}}+O\left(q^{5}\right)$ which is exactly the result obtained for the five-dimensional perturbative Einstein-Maxwell black hole [25]. For small $\beta$ the magnetic moment is negative. We analyze this result below, when we comment on the gyromagnetic ratio.

The dimensional gyromagnetic ratio for a given system defined in Eq. (26) is twice the ratio of the magnetic moment divided by the charge to the angular momentum divided by the mass. It has the value 1 for a classical body with uniform mass and uniform charge distribution rotating about an axis of symmetry. For an electron it has the value of 2.00 plus small quantum corrections, and for the proton 5.59 and neutron has the value 5.59 and -3.826 , respectively. From Eq. (26) we see that the perturbative parameter $q$ and the parameters $\beta$ and $\nu$ modify the gyromagnetic ratio of asymptotically flat five-dimensional charged rotating black holes as compared to the uncharged extremal Myers-Perry black holes. We want to study in more detail this modification of the gyromagnetic ratio when one varies the Born-Infeld parameter $\beta$, see Fig. 3. From the figure we find that the gyromagnetic ratio $g$ increases with increasing $\beta$, and in the limit $\beta \rightarrow \infty$, the gyromagnetic ratio reduces to $g=3+\frac{q^{2}}{12 \nu^{4}}-\frac{q^{4}}{144 \nu^{8}}+O\left(q^{6}\right)$ which is exactly the result obtained for the five-dimensional perturbative Einstein-Maxwell black hole [25]. Now, from Fig. 3 one finds that for some low value of $\beta$ the gyromagnetic ratio is zero, and then turns negative. This change 


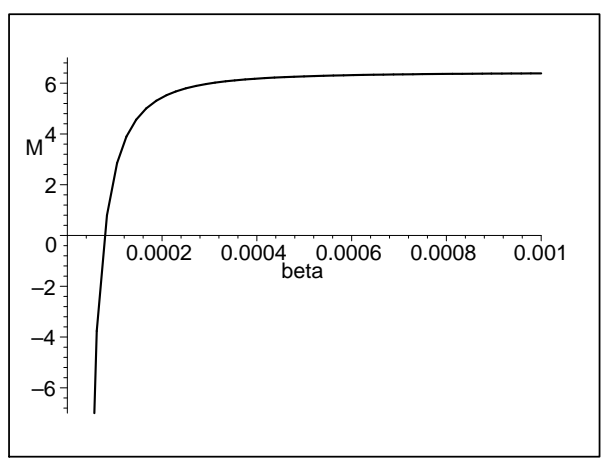

FIG. 1: The black hole mass $M$ versus the Born-Infeld parameter $\beta$ for $\nu=1.16$ and $q=0.09$.

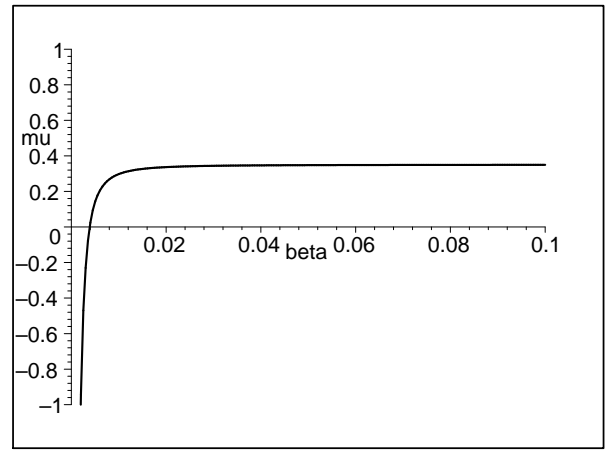

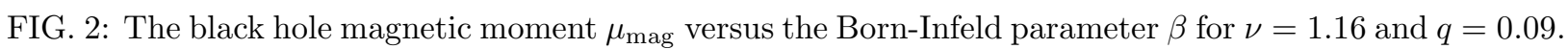

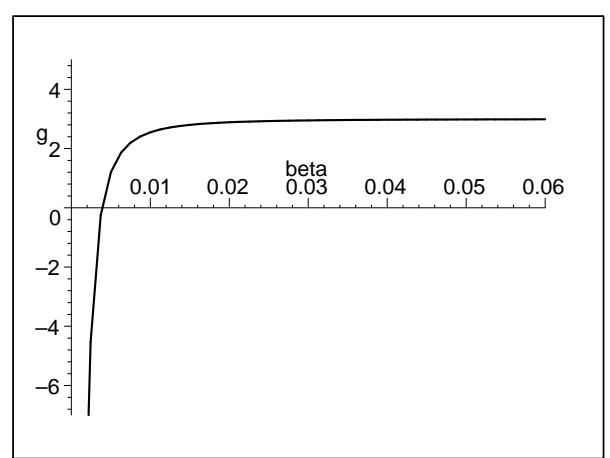

FIG. 3: The black hole gyromagnetic ratio $g$ versus the Born-Infeld parameter $\beta$ for $\nu=1.16$ and $q=0.09$.

of sign comes from a perturbative $q^{2}$ term, and thus the result might not hold for the full exact solution. On the other hand the result is sufficiently intriguing that deserves some attention. One can speculate that it is at least qualitatively correct, and perhaps expected. Let us see why. It is known that the Born-Infeld theory is different from Maxwell's theory when the electromagnetic fields are very strong. The Born-Infeld theory gives a finite total energy $E$ for the field around a point particle with charge $q_{\text {particle }}$, indeed $E \simeq \sqrt{q_{\text {particle }}^{3} \beta}$. It also gives an effective radius $r_{0}$ for the charge distribution, $r_{0}=\sqrt{\frac{q_{\text {particle }}}{\beta}}$. Curiously, the reversal of the gyromagnetic ratio $g$ in 
Fig. 3 (concomitant to the reversal of the magnetic dipole moment $\mu_{\text {mag }}$ in Fig. 2) happens when $r_{0}=\sqrt{\frac{Q}{\beta}}$ is of the order or larger than the horizon radius $r_{H}$. Indeed, for the values of $\nu$ and $q$ used in the figures, one finds $r_{0}=8.79$ and $r_{H}=1.65$. This reversal could then be interpreted as follows. For large $\beta$ much of the electrical charge is distributed in a point-like manner, as in Maxwell's theory. For small $\beta$ the charge distribution is extended, and for sufficiently small $\beta$ it even extends outside the horizon. It is known that an object with magnetic moment $\vec{\mu}_{\text {mag }}$ placed on a magnetic field $\vec{B}$ suffers a torque given by $\vec{\mu}_{\text {mag }} \times \vec{B}$. A black hole with magnetic moment is also subjected to this kind of torque. So, for large $\beta$, i.e., the point like case, when a magnetic field is applied to the black hole spacetime the resultant torque on the black hole tends to rotate it in the expected sense, and thus the magnetic dipole moment $\mu_{\text {mag }}$ and $g$ are positive, On the other hand, when $\beta$ is small, i.e., the charge distribution outside the black hole case, it is the region external to the black hole horizon that is effectively charged and it is this very region that upon application of a magnetic field tends to rotate in the expected sense. So here, the black hole rotates in the opposite sense, giving a negative magnetic dipole moment $\mu_{\text {mag }}$ and thus a negative gyromagnetic ratio $g$.

The horizon radius $r_{H}$ in Eq. (27) has the unexpected feature that it does not depend on $\beta$ at least up to 4th order in the charge. Up to this order $r_{H}$ is equal to the Einstein-Maxwell case [25].

\section{THE MASS FORMULA}

Define $\xi$ as the timelike Killing vector and $\eta_{k}, k=1,2$, as the two azimuthal Killing vectors. The two equal horizon constant angular velocities $\Omega$ can then be defined by imposing that the Killing vector field

$$
\chi=\xi+\Omega \sum_{k=1}^{2} \epsilon_{k} \eta_{k},
$$

is null on the horizon and orthogonal to it as well. This yields,

$$
\Omega=\frac{1}{2 \nu}-\frac{q^{2}}{24 \nu^{5}}-\frac{q^{4}\left(5 \nu^{2} \beta^{2}-1\right)}{1440 \beta^{2} \nu^{11}}+O\left(q^{6}\right)
$$

The 3-area of the horizon $A_{H}$ and the electrostatic potential at the horizon $\Phi_{H}$ are given by

$$
\begin{gathered}
A_{H}=8 \pi^{2} \nu^{3}+O\left(q^{6}\right) . \\
\Phi_{H}=\frac{q}{4 \nu^{2}}+\frac{q^{3}\left(20 \beta^{2} \nu^{2}\right)}{1440 \beta^{2} \nu^{8}}+O\left(q^{5}\right) .
\end{gathered}
$$


The surface gravity $\kappa$ is defined by $\kappa^{2}=-\frac{1}{2}\left(\nabla_{\mu} \chi_{\nu}\right)\left(\nabla^{\mu} \chi^{\nu}\right)$. Taking into account the conserved quantities obtained in the last section, one can check that these quantities satisfy the Smarr mass formula up to 4 th order [8]. Indeed, in general the formula is

$$
M=\frac{3 \kappa_{s g} A_{H}}{16 \pi G_{D}}+3 \Omega J+\Phi_{H} Q-\frac{\beta}{2} \frac{\partial M}{\partial \beta} .
$$

For an extremal solution with $\kappa=0$, the Smarr mass formula reduces to

$$
M=3 \Omega J+\Phi_{H} Q-\frac{\beta}{2} \frac{\partial M}{\partial \beta} .
$$

Taking into account that the mass $M$ of the black holes is given by Eq. (22), one can determine the last term in (33), and find

$$
M=3 \Omega J+\Phi_{H} Q+\Phi_{\beta} Q^{4}
$$

where

$$
\Phi_{\beta}=-\frac{\beta}{2 Q^{4}} \frac{\partial M}{\partial \beta}=-\frac{1}{1920} \frac{1}{\pi^{3} \nu^{8} \beta^{2}} .
$$

\section{CONCLUSIONS}

In conclusion, we have presented a new class of perturbative charged rotating black hole solutions in five dimensions in the presence of a nonlinear Born-Infeld gauge field. This class of solutions is restricted to the extremal black holes with equal angular momenta. At infinity, the metric is asymptotically locally flat. Our strategy for obtaining these solutions was through a perturbative method up to the 4 th order for the perturbative parameter $q$. We have started from rotating MyersPerry black hole solutions [17] in five dimensions, and then studied the effects of adding a charge parameter to the solutions. We have calculated the conserved quantities of the solutions such as mass, angular momentum, electric charge, magnetic moment, gyromagnetic ratio, and horizon radius. We found that the Born-Infeld parameter $\beta$ modifies the values of all the physical quantities, except the horizon radius, relative to the corresponding Einstein-Maxwell five dimensional rotating solutions. For large $\beta$ the solutions reduce to the perturbative rotating Einstein-Maxwell solutions [25], as we expected. We also speculated on what might happen for these solutions in the strong electromagnetic regime, i.e., when $\beta$ is small. The generalization of the present work to all higher dimensional is quite an interesting subject which will be addressed elsewhere. 


\section{Acknowledgments}

The support of the Fundação para a Ciência e a Tecnologia of Portugal Project PTDC/FIS/098962/2008 and PEst-OE/FIS/UI0099/2011 is gratefully acknowledged. M. Allaverdizadeh is supported by a FCT grant. The work of A. Sheykhi has been supported financially by Research Institute for Astronomy and Astrophysics of Maragha (RIAAM), Iran.

[1] F. Tangherlini, Nuovo Cimento 27, 636 (1963).

[2] M. Bañados, C. Teitelboim and J. Zanelli, Phys. Rev. D 49, 975 (1994);

G. A. S. Dias, S. Gao and J. P. S. Lemos, Phys. Rev. D 75, 024030 (2007).

[3] A. Garcia, H. Salazar and J. F. Plebanski, Nuovo. Cimento 84, 65 (1984);

N. Breton, Phys. Rev. D 67, 124004 (2003).

[4] D. L. Wiltshire, Phys. Rev. D 38, 2445 (1988);

H. P. de Oliveira, Class. Quant. Grav. 11, 1469 (1994).

[5] M. Aiello, R. Ferraro and G. Giribet, Phys. Rev. D 70, 104014 (2004).

[6] T. Tamaki, Journ. Cosm. Astropart. Physics JCAP 0405, 004 (2004)

[7] T. K. Dey, Phys. Lett. B 595, 484 (2004).

[8] S. Gunasekaran, D. Kubiznak and R. B. Mann, Journal of High Energy Physic JHEP 11, 110 (2012).

[9] M. H. Dehghani and S. H. Hendi, Int. J. Mod. Phys. D 16, 1829 (2007).

[10] M. Aiello, R. Ferraro and G. Giribet, Phys. Rev. D 70, 104014 (2004);

M. H. Dehghani, N. Alinejadi and S. H. Hendi, Phys. Rev. D 77, 104025 (2008).

[11] R. G. Cai, D. W. Pang and A. Wang, Phys. Rev. D 70, 124034 (2004).

[12] I. Stefanov, S. S. Yazadjiev and M. D. Todorov, Phys. Rev. D 75, 084036 (2007);

I. Stefanov, S. S. Yazadjiev and M. D. Todorov, Mod. Phys. Lett. 22, 1217 (2007).

[13] R. Yamazaki and D. Ida, Phys. Rev. D 64, 024009 (2001).

[14] T. Tamaki and T. Torii, Phys. Rev. D 62, 061501 (2000);

T. Tamaki and T. Torii, Phys. Rev. D 64, 024027 (2001);

G. Clément and D. Gal'tsov, Phys. Rev. D 62, 124013 (2000).

[15] S. S. Yazadjiev, Phys. Rev. D 72, 044006 (2005).

[16] A. Sheykhi, N. Riazi and M. H. Mahzoon, Phys. Rev. D 74, 044025 (2006);

A. Sheykhi, N. Riazi, Phys. Rev. D 75, 024021 (2007);

M. H Dehghani, S. H. Hendi, A. Sheykhi and H. Rastegar Sedehi, Journ. Cosm. Astropart. Physics JCAP 0702, 020 (2007);

A. Sheykhi, Int.J. Mod.Phys. D 18, 25 (2009);

A. Sheykhi, Phys. Lett. B 662, 7 (2008). 
[17] R. C. Myers and M. J. Perry, Ann. Phys. (N.Y.) 172, 304 (1986).

[18] G. W. Gibbons, H. Lü, D. N. Page and C. N . Pope, J. Geom. Phys. 53, 49 (2005).

[19] A. N. Aliev, Phys. Rev. D 74, 024011 (2006).

[20] A. N. Aliev, Mod. Phys. Lett. A 21, 751 (2006);

A. N. Aliev, Class. Quant. Gravit. 24, 4669 (2007);

A. Sheykhi, Phys. Rev. D 77, 104022 (2008).

[21] A.N. Aliev and D.K. Ciftci, Phys. Rev. D 79, 044004 (2009).

[22] J. Kunz, F. Navarro-Lerida and A. K. Petersen, Phys. Lett. B 614, 104 (2005).

[23] J. Kunz, F. Navarro-Lerida and J. Viebahn, Phys. Lett. B 639, 362 (2006).

[24] J. Kunz, F. Navarro-Lerida and E. Radu, Phys. Lett. B 649, 463 (2007);

A. N. Aliev, Phys. Rev. D 75, 084041 (2007);

H. C. Kim and R. G. Cai, Phys. Rev. D 77, 024045 (2008);

Y. Brihaye and T. Delsate, Phys. Rev. D 79, 105013 (2009);

A. Sheykhi, M. Allahverdizadeh, Phys. Rev. D 78, 064073 (2008).

[25] F. Navarro-Lerida, Gen. Relat. Gravit. 42, 2891 (2010).

[26] M. Allahverdizadeh, J. Kunz and F. Navarro-Lerida, Phys. Rev. D 82, 024030 (2010).

[27] M. Allahverdizadeh, J. Kunz and F. Navarro-Lerida, Phys. Rev D 82, 064034 (2010). 